\title{
Investigating the Use and Performance of a Three-wheeler used in Inner City Transportation in Sub-Saharan Africa
}

\author{
${ }^{* 1}$ Oluwaseun K. Ajayi, ${ }^{2}$ Oluleke Oluwole, ${ }^{1}$ Adebayo Fadairo, and ${ }^{3,4}$ Abiola J. Adeyi \\ ${ }^{1}$ Department of Mechanical Engineering, Obafemi Awolowo University, Ile-Ife, Nigeria \\ ${ }^{2}$ Department of Mechanical Engineering, University of Ibadan, Nigeria \\ ${ }^{3}$ Department of Mechanical Engineering, Ladoke Akintola University, Ogbomoso, Nigeria \\ ${ }^{4}$ Forestry Research Institute of Nigeria, Ibadan, Nigeria \\ okajayi@oauife.edu.ng | ooluwole@gmail.com l afadairo@oauife.edu.ng | adeyi.abiola@yahoo.com
}

Received: 08-SEP-2021; Reviewed: 20-SEP-2021; Accepted: 21-SEP-2021

http://dx.doi.org/10.46792/fuoyejet.v6i3.694

\begin{abstract}
The dominance of three-wheelers in inner city transportation cannot be overrated, because it is presumed to be safer compared to two-wheel drives. It is an intermediary between the motor cycles and taxis, as it is efficient for both passengers and goods transportation. However, as manufacturers introduce newer brands into the market, the impact relating to mechanical functioning is usually overlooked. This study was designed to evaluate the performance of the transmission system of the three-wheeler. In order to gather background information on the three-wheeler and its users, a questionnaire was administered to thirty (30) drivers. A Bajaj engine was selected because of the orientation of the output shaft and mounted on a test-rig equipped with a dynamometer. Output speed (OS), output torque (OT), fuel consumed and time taken under five loading conditions namely; No Load, 120W, 800W, 920W and 1600W corresponding to idle, downhill, level ground and overload/uphill conditions were recorded. However, it was discovered that $60 \%$ of the drivers were less than 40 years old, while the remaining $40 \%$ were adults. The output speed and torque for No-load and downhill, likewise medium load and level ground are similar, but the uphill was different. The results showed that Bajaj and TVS brands are used, Bajaj being the oldest. Also, there is significant variation in engine output with loading and terrain navigation with associated noise as load increases. The manual transmission fitted with the engine seems unable to properly transmit engine output from the shafts. A better transmission is therefore recommended.
\end{abstract}

Keywords- Dynamometer, Output speed, output torque, three-wheeler

\section{INTRODUCTION}

T he use of three-wheelers otherwise known as Keke Marwa in Nigeria has come to stay. Many find it more comfortable and safer than motorcycles, so it is allowed in areas where the use of motorcycles is restricted. Because of its slim shape, it is easier to maneuver (Radhakrishna et al., 2015), making it easier to be driven in busy areas especially in the clumsy markets (Machanda, 2016) are typical of most markets in Nigeria.

Gore et al., (2015) believed that the demand for threewheelers would increase rapidly in the nearest future, as it is the most affordable means of transportation in many countries (Asghar et al., 2016). The three-wheeler has been very useful serving transportation needs as shuttles within hospitals, educational institutions, industries and other large premises (Joshi et al., 2008). A survey carried out by Lukic et al., (2007) in India helped to derive a usage pattern for the three-wheeler by gathering information through the use of GPS data and a physical survey of three-wheeler drivers.

Data gathered from dynamometer test conducted for 40 Indian three-wheelers with three different fuel-engine combinations operating on the Indian Drive Cycle (IDC) were used to develop velocity-acceleration lookup tables for fuel consumption, Carbon oxide emissions, hydrocarbons, nitrogen oxides, and fine particulate matters for each fuel-engine combination (Grieshop et al., 2012).

${ }^{*}$ Corresponding Author

Section C- MECHANICAL ENGINEERING \& RELATED PHYSICAL SCIENCES Can be cited as:

Ajayi O., Oluwole O., Fadairo A. and Adeyi A. (2021): Investigating the Use and Performance of a Three-wheeler used in Inner City Transportation in SubSaharan Africa, FUOYE Journal of Engineering and Technology (FUOYEJET), 6(3), 39-42. http://dx.doi.org/10.46792/fuoyejet.v6i3.694
Also, emission characteristics of the Indian Drive Cycle and a Modified Indian Drive Cycle were compared by Adak et al., (2016). Furthermore, to investigate the output of hybrid three-wheelers with the conventional ICE engine models, it is better to compare the power-train performance (Abu et al., 2016) used for its speed and torque transmissions (Sanmugan, 2016).

Investigation into the means of transportation is not new (Ehinmowo et al., 2021 and Zayet et al., 2021). Threewheelers are known to be noisier than other passenger vehicles. In addition, the load carrying capacity seems to be poor because the speed usually low when loaded to full capacity or it is carrying goods with some weights. This work was carried out to investigate the usage and performance of a three-wheeler called Keke Napep under varying loading capacity, in order to ascertain areas of drudgery and the causes with the aim of suggesting improvements or adaptations for the subsequent upgrades for the vehicle.

\section{Methodology \\ 2.1 PRELIMINARY RESEARCH/ INFORMATION}

Preliminary research aimed at extracting useful information from three-wheeler riders was carried out. This was done by administering a questionnaire to them where there is a cluster of them. Three keke-NAPEP parks in Ibadan, Oyo state were selected for this survey with a total of 30 respondents.

\subsection{MATERIALS}

The materials used for the experiment were; A Bajaj brand internal combustion engine, a digital tachometer for speed measurement, a dynamometer for torque measurement, and a test rig for engine performance testing. The test rig was designed taking into consideration Stability, Vibration damping, Mobility, 
Ease of attaching measuring devices and equipment as well as fuel tank, Easy assembly, and Compatibility. The dynamometer used for data collection has been fitted with braking system which tends to restrict the rotation of the wheels, thereby serving as load or driving conditions. The specifications of these loads are $120 \mathrm{~W}$, $800 \mathrm{~W}, 920 \mathrm{~W}$ and $1600 \mathrm{~W}$ adequately describing the driving conditions of the three-wheeler loading and its terrain navigation performance.

\subsection{Methods}

The Bajaj RE three-wheeler engine was placed on the test rig. The output shaft of the engine where the axle is usually connected to was the point of measurement. It is the point where the wheels are usually attached, so this was used as the point of the gear output parameter measurement. The dynamometer was then connected to the output shaft to measure the output power and energy outputs. The tachometer was placed with the axis of the infra-red ray intersecting with the reflective tape attached to the pulley of the dynamometer. The USB data cable was then connected from the dynamometer to the HP laptop output power and energy data acquisition. Likewise, the data cable of the tachometer was also connected to the Dell Laptop for output speed data acquisition.

An improvised starter was used for the engine cranking by replacing the engine starter linkage with a shorter one with only one linkage. This linkage was pulled like the originally fitted linkage to start the engine. A measured quantity of fuel was put into the tank attached to the engine. The fuel container was attached to the carburetor the power meter was connected to the output shaft via a v-belt. The tachometer was placed close to the output pulley and a reflective tape was attached to the tip of the pulley. The tachometer and dynamometer were attached to the points where they would measure the speed, power and Energy outputs of the engine.

The engine was started while the gear was in the neutral position. Gear 1 was engaged and the throttle was opened gradually to full throttle which increases the output speed. The energy released (ER) from the engine, output power (OP), and output speed (OS) progressions were recorded for this gear ratio. These procedures were followed for the other transmission ratios and the loading conditions. The time taken to complete the test (gear one to gear four) and the quantity of fuel consumed for each experiment under the various loads were also recorded.

\section{Results And Discussions \\ 3.1 Preliminary SuRVEY}

Some information was extracted from three-wheeler drivers to ascertain some peculiarities of the vehicle usage. The results are presented in Fig 1-3.

It was discovered that the Frequency of routine servicing is monthly and the Fuel tank Capacity is 5ltrs for Bajaj while it's 8-10ltrs for TVS. The implication of this is that the TVS brand can run for more time before refueling. The result of the survey showed that $66.37 \%$ use the Bajaj brand while $33.33 \%$ use the TVS. Also, $60 \%$ of the three- wheeler drivers were in their 30 s and $75 \%$ of the oldest (those above 40 years) use the Bajaj brand, while the rest $25 \%$ use the TVS brand. The middle-aged drivers in their 40 s have the range of $52 \%$ using TVS brand while $48 \%$ uses the Bajaj brand.

Servicing of the three-wheeler is done bi-monthly for both vehicle brands. However, the recommended manufacturer's maintenance schedule is to replace and filter after every $500-750 \mathrm{~km}$ operations. TVS brands were fitted with both kick-starters and manual cranking levers, to start the engine while $80 \%$ of the Bajaj brands have both means of starting the engine. The remaining $20 \%$ of the Bajaj brand uses only the manual means of starting the engine which was those older than two (2) years.

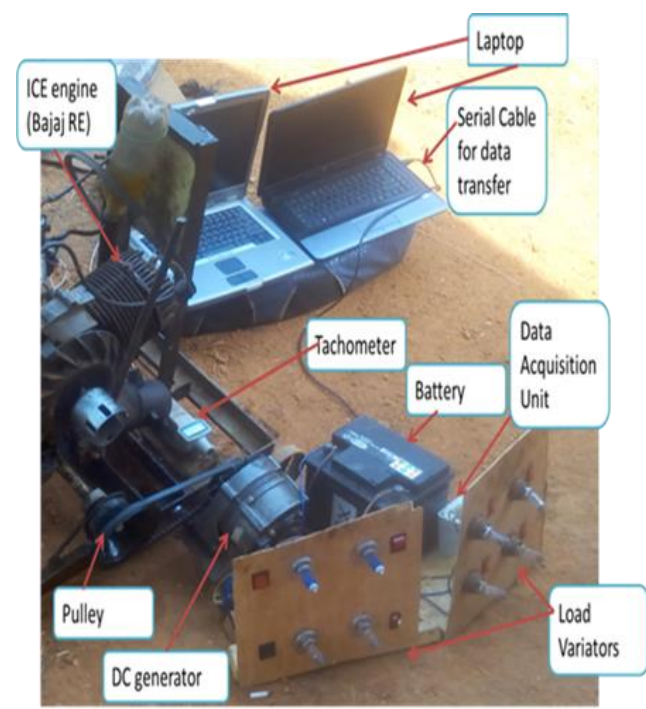

Plate 1: Manual transmission testing rig setup

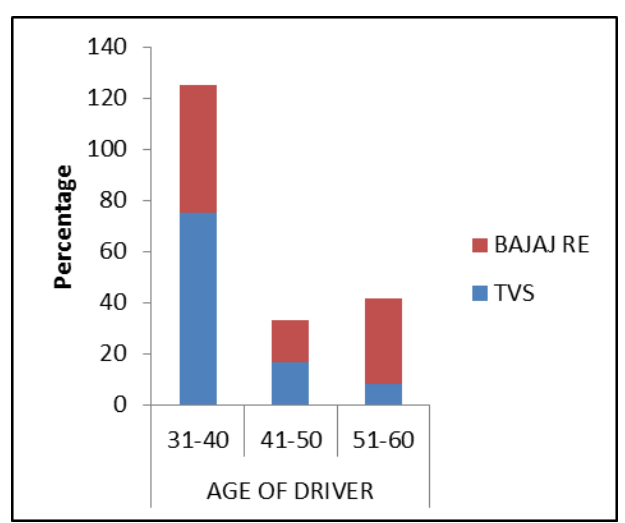

Fig. 1: Age of distribution of drivers using Bajaj versus TVS

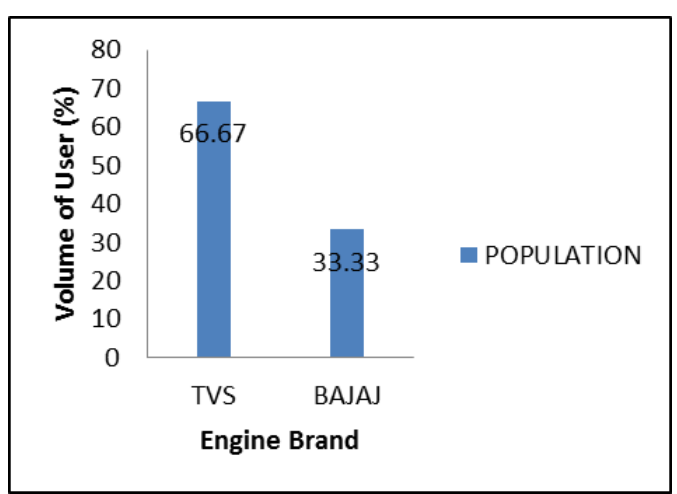

Fig. 2: Percentage of Bajaj users versus TVS users 


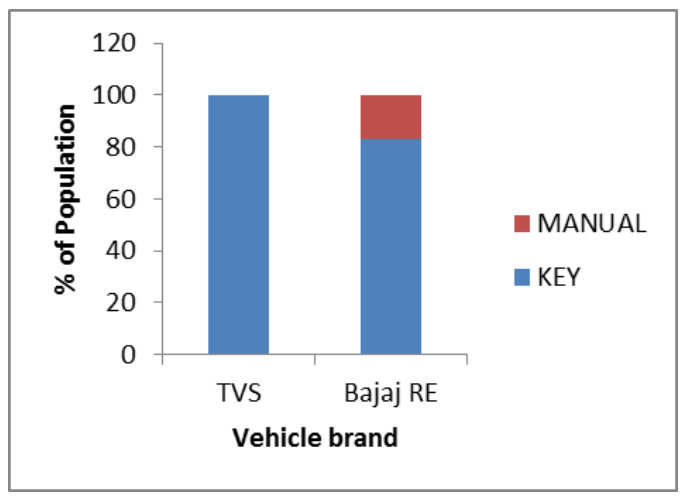

Fig. 3: Type of kick-start fitted with Bajaj versus kick-start fitted with TVS

\subsection{Engine Performance Characteristics}

The manual transmission works directly with the engine. The engine supplies the torque needed to overcome load because the gears act as torque and speed converters for the engine. The performance result of Speed (OS) and torque (OT) are presented in Figs 4-9 for the loading conditions.

At no load in Fig. 5, the graph presented a lower slope than others. In Figs $5 \& 6$, there was a close match between the plot of no load and load 120W). This shows that the loading condition (driving condition) is relatively close, so there was a close behaviour in the engine output. At load $1600 \mathrm{~W}$, the speed variation was rather shorter than other loading conditions. This depicts that the engine might be overloaded. The maximum speed was therefore attained in less time compared to others.

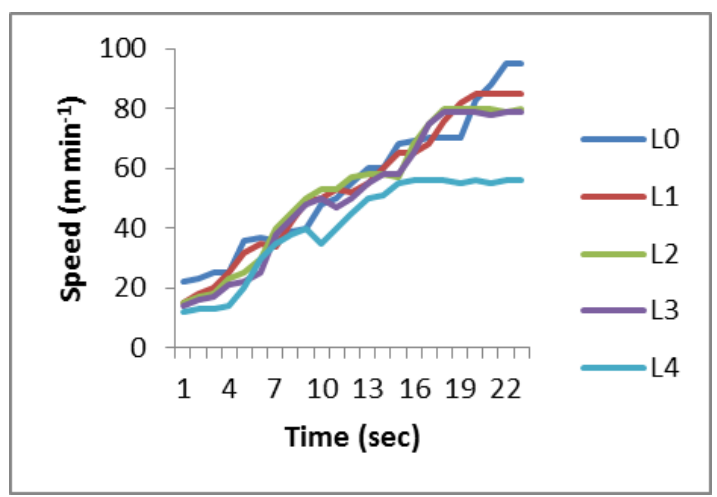

Fig. 4: Speed graph for all loading conditions

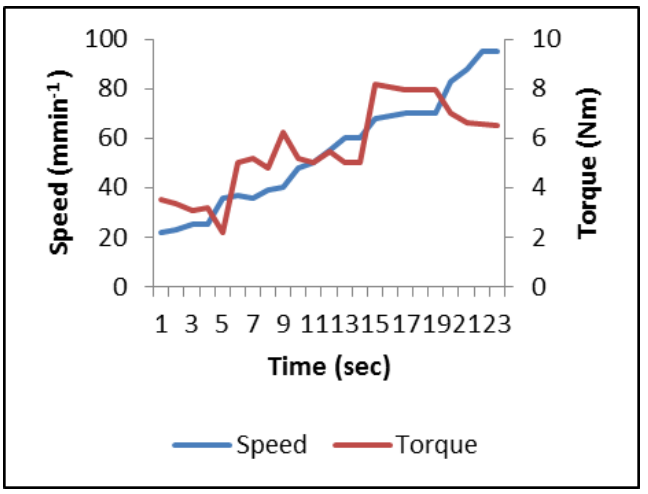

Fig. 5: Speed vs Torque (MT at No Load)

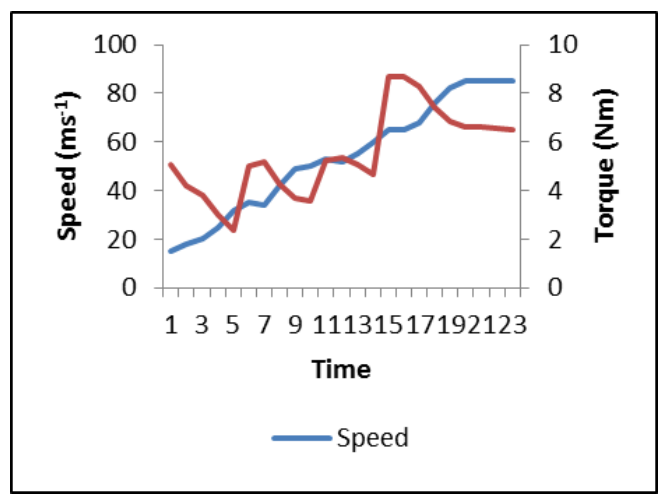

Fig. 6: Speed vs Torque (MT at 120W)

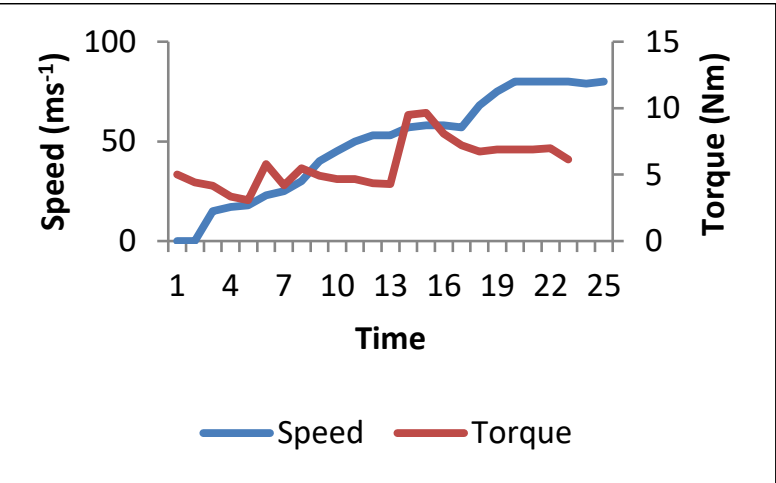

Fig. 7: Speed vs Torque (MT at $800 \mathrm{~W}$ )

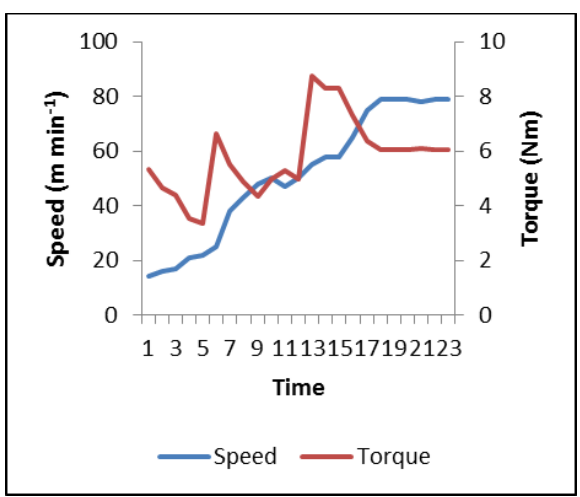

Fig. 8: Speed vs Torque (MT at 920W)

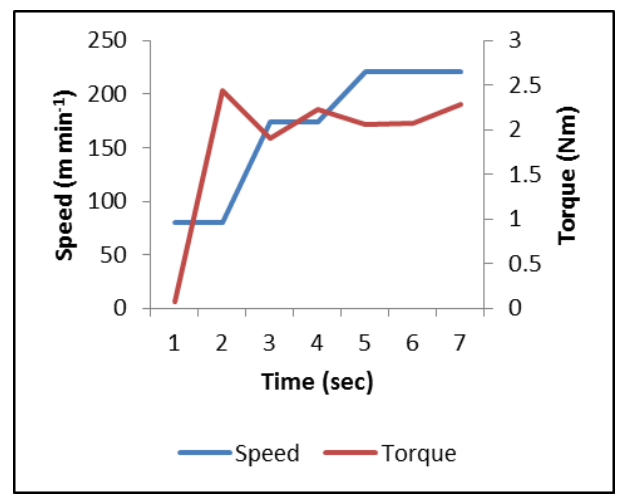

Fig. 9: Speed vs Torque (MT at 1600W)

The choice of bulbs used as loads effectively loaded the engine as seen in all the graphs, because it was the reason for the various variations presented. The highest load $1600 \mathrm{~W}$ was significant in all the readings shown, depicting uphill traveling. Average fuel consumption was 0.17 litres for each driving cycle which is when running the engine through all the 
gear transmissions for a particular load. The time taken to complete each driving cycle was the same in the first three loading conditions while the highest loading condition was shorter depicting overload.

\section{Conclusions and Recommendations}

The following were observed and recommended;

i. Two brands of three-wheeler are majorly used in Nigeria; the Bajaj Re which is the oldest and the TVS.

ii. $60 \%$ of the drivers were less than 40 years old, while the remaining $40 \%$ are adults and use the Bajaj RE brand.

iii. Speed variations downhill and level ground corresponding to low and medium (intermediate) loading conditions does not show much variation for each transmission.

iv. The torque fluctuates about a mean.

v. For further studies, a continuously variable transmission can be retrofitted into the transmission to eliminate the drudgery in gear change, increase torque and reduce fuel consumption.

\section{REFERENCES}

Abu, M., Denman, B. \& Surgenor, B., (2010). A Study of Fuel Cell Hybrid Auto Rickshaws Using Realistic Urban Drive Cycles, 4(1), 225-229.

Adak, P., Sahu, R. \& Elumalai, S.P., (2016). Development of emission factors for motorcycles and shared auto-rickshaws using real-world driving cycle for a typical Indian city. Science of the Total Environment, 544, 299-308. http://dx.doi.org/10.1016/j.scitotenv.2015.11.099.

Asghar, A.M., Bhatti, A.I., Ahmed, Q. and Izhar, T. (2016). Design of Efficient Electric Auto Rickshaw. Proceedings of the 18th International Conference on Electrical Engineering, Computer Science and Technology, Istanbul, Turkey.

Ehinmowo A. B., Ariyo O. O., Ohiro O. A., Fajemidupe O. T. and Salam K. K. (2021). An improved data-driven model for the prediction of minimum transport condition for sand transporting multiphase flow systems. FUOYE Journal of Engineering and Technology, 6(1), $103-107$.

Gore, M.M., Ronge, B.P. \& Misal, N.D. (2015). A review paper on design and analysis of system of three-wheeler. International Journal of Application or Innovation in Engineering \& Management (IJAIEM), 4(5), 77-80.

Grieshop, A.P., Boland, D., Reynolds, C.C.O., Gouge, B., Apte, J.S., Rogak, S.N., \& Kandlikar M., (2012). Modeling air pollutant emissions from Indian auto-rickshaws: Model development and implications for fleet emission rate estimates. Atmospheric Environment, 50, 148-156. http://dx.doi.org/10.1016/j.atmosenv.2011.12.046.

Joshi, M., D'Silva, B., Subhan, I. \& Kumar A. (2008). Autorickshaw Vehicles: A National out-of-hospital care plan for India. Proceedings of ICEM 2008 Scientific Abstract Program.

Lukic,S. M., Mulhall, P., Choi, G., Naviwala, M., Nimmagadda, S. \& Emadi, A. (2007). Usage pattern development for threewheel auto rickshaw taxis in India. VPPC 2007 Proceedings of the 2007 IEEE Vehicle Power and Propulsion Conference, 610-616. doi: 10.1109/VPPC.2007.4544195

Manchanda, M. (2016). Auto-rickshaw: Yesterday, today and tomorrow. Business Standard. Available at: http://www.business-standard.com/article/beyond- business/auto-rickshaw-yesterday-today-and-tomorrow116072500265_1.html [Accessed February 6, 2017].

Radhakrishna, K., Srinivasa Rao, S. \& Sudhakara Rao, B. (2015). Design and Analysis of Dump Body on Three Wheeled Auto Vehicle. International Journal on Cybernetics \& Informatics, $4(2), 65-73$.

Sanmugan, R. (2016). What is continuously variable transmission? How does it work? Retrieved from https://www.quora.com/What-is-continuously-variabletransmission-How-does-it-work. [Accessed September, 2021].

Zayet T. M. A., Ismail M. A., Varathan K. D., Noor R. M. D., Chua H. N., Lee A., Low Y. C. and Singh S. K. J. (2021). Investigating transportation research based on social media analysis: a systemic mapping review. Scientometrics, 125, $6383-6421$ 